\title{
Management of Displaced Intra-Articular Calcaneal Fractures: A Comparative Study of Open and Minimally Invasive Surgery
}

\author{
Amirul Islam ${ }^{1}$, Charles Mcdonald ${ }^{2}$, Ahmed Aljawadi ${ }^{1}$, Noman Niazi $^{1}$, Anand Pillai ${ }^{1}$ \\ 1. Trauma and Orthopaedics, Wythenshawe Hospital, Manchester, GBR 2. Surgery, University of Manchester, \\ Manchester, GBR
}

Corresponding author: Amirul Islam, doctor_amirul@yahoo.com

\section{Abstract \\ Objectives}

The ideal treatment of displaced intra-articular calcaneal fractures continues to be a subject of debate. The aim of the study was to compare the radiological outcome, cumulative radiation exposure, surgical time, time to surgery, wound healing times and cost involved in minimally invasive surgery (MIS) and open reduction internal fixation (ORIF) for calcaneal fractures.

\section{Methods}

This was a retrospective study of 39 calcaneum operated in our unit during 2012 to 2019, of which 20 had undergone ORIF and 19 had been operated upon following MIS.

\section{Results}

A total of 39 calcanea (37 patients) were operated, of which 20 had open procedure and 19 had MIS procedure, including one bilateral surgery in each group. Mean age of the patients in the MIS group was 42.18 years (range: $15-68$ years) and that of the patients in the open group was 43 years (range: $21-75$ years). Of the fractures, $53.84 \%(n=21)$ was Sanders type III, 28.20\% $(n=11)$ was type II and $17.94 \%(n=7)$ was type IV. There was no statistically significant difference in the mean correction of Bohler's angle and Gissane's angle between the groups. The mean cost for implant used for each open procedure was $£ 882.79$, and the implant cost for each MIS procedure was £142.89. Mean utilisation of cumulative X-ray dose was significantly higher in MIS ( $0.764 \mathrm{mGy})$ in comparison to open surgery $(0.392 \mathrm{mGy})$. The average surgical time for MIS was 64.9 minutes and that of open surgery was 106.3 minutes. Average waiting time for MIS was 6.6 days and that for ORIF was 9.8 days. Wound healing was quicker (average 13.4 days) in MIS than ORIF (average 17.2 days). All these differences were statistically significant.

\section{Conclusions}

Received 07/01/2020

Review began $07 / 08 / 2020$ Review ended 07/21/2020 Published 08/04/2020

\section{(c) Copyright 2020}

Islam et al. This is an open access article distributed under the terms of the Creative Commons Attribution License CC-BY 4.0., which permits unrestricted use, distribution, and reproduction in any medium, provided the original author and source are credited.
Minimally invasive calcaneal fracture surgery is quicker and cheaper and can be performed earlier. It is associated with early wound healing, although it requires higher cumulative radiation dose.

Categories: Radiology, Orthopedics, Trauma

Keywords: displaced intra-articular calcaneal fracture, cost analysis, radiological outcome, sinus tarsi approach, minimally invasive surgery

\section{Introduction}

Calcaneal fractures (CFs) account for more than $60 \%$ of tarsal bone fractures and around $2 \%$ of all fractures [1]. This can be a debilitating fracture and is most common in those who are economically active [2]. Ideal management of these fractures remains controversial. Open reduction internal fixation (ORIF) is usually performed utilising an extensile lateral approach, and plate and screws are used for internal fixation. However, this has been reported with $15 \%$ to $25 \%$ of wound-related complications or flap necrosis [3].

Current evidence suggests that less invasive surgical options could achieve equally satisfactory radiological outcomes compared to open techniques [3,4]. We have analysed pre- and post-operative radiological parameters such as Bohler's angle, critical angle of Gissane, and calcaneal height and width. We have also investigated costs involved in the fixation method to find out any difference. In our study we also looked into the cumulative radiation exposure during fluoroscopy, time to surgery, duration of surgery, wound healing and other complications for both minimally invasive surgery (MIS) and open CFs.

\section{Materials And Methods}




\section{Cureus}

A retrospective review was undertaken of all patients who underwent either MIS or ORIF for CFs in our unit from 2012 to 2019. The type of surgery was preference and practice of different foot and ankle (F\&A) units of the same trust. Surgery was performed in all cases by a consultant F\&A surgeon. A total of 37 patients were identified. Data were collected from a number of sources including electronic patient records, discharge summaries, clinic letters and Picture Archiving and Communication System (PACS).

Outcome measures included a number of key radiological markers such as Bohler's angle, Gissane's angle, and calcaneal width and height. Pre- and post-operative measurements were calculated to find out the amount of correction achieved and their statistical significance.

We also looked into the cost of implants involved in the fixation method and the cumulative radiation dose while using fluoroscopy during the procedure. Cost of implant was obtained from hospital procurement, and fluoroscopic dose was obtained from the PACS record. Surgical time was calculated from the Operating Room Management Information System (ORMIS) records.

All patients had isolated injuries, and soft tissue status was carefully assessed. All patients had plain radiograph as well as computed tomography (CT) scan (Figure 1), and fractures were classified according to Sanders [5]. Initial management included below knee slab in a neutral position, leg elevation, analgesia and prophylaxis for venous thromboembolism. Surgery was performed once the soft tissue condition permitted as evident by the reduction of swelling and appearance of skin wrinkles.
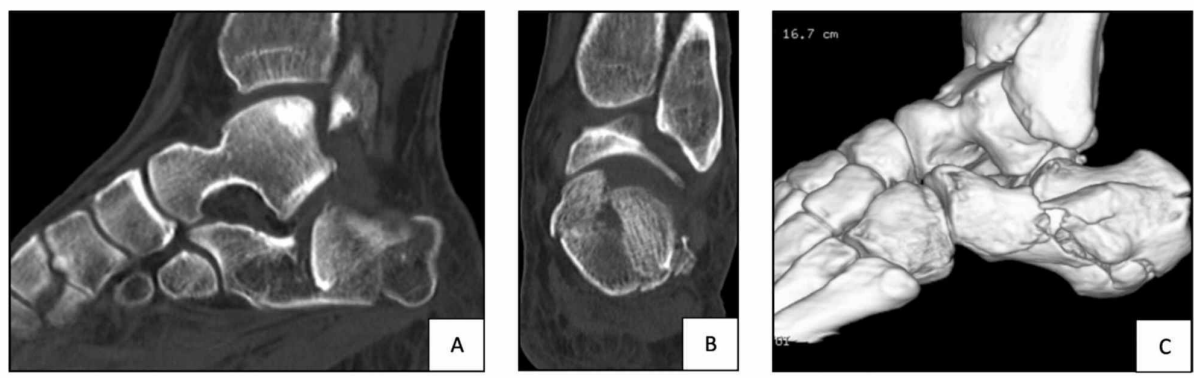

\section{FIGURE 1: Sagittal (A), coronal (B) and three-dimensional reconstruction (C) CT scan of the calcaneal fracture treated by MIS fixation.}

*Same patient's intra-operative photograph and fluoroscopic images are shown in Figures 2, 3, respectively.

MIS, minimally invasive surgery

Sanders classification [5] is based on the number of articular fragments seen on coronal CT scans at the widest point of the posterior facet of the calcaneum. It has been shown to be useful in determining treatment as well as prognosis [5].

All patients were operated in a lateral position under tourniquet control after receiving prophylactic intravenous antibiotics. For open surgery cohort $(n=20)$, extensile lateral approach was followed with meticulous tissue dissection to raise full-thickness flap. After open reduction, appropriate size locking plate (Zimmer Biomet A.L.P.S, Swindon, UK) was used for fixation. For the MIS cohort $(\mathrm{n}=19)$, limited sinus tarsi approach was used (Figure 2). The displaced intra-articular fracture was reduced with the help of small osteotomes and elevators under fluoroscopic guidance and findings from the pre-operative study of CT scan. To hold the articular reduction, 4-mm cannulated screws were used. For extra-articular reduction, especially to get the calcaneum out to length and to correct the varus deformity, a $3.2-\mathrm{mm}$ wire was used for joy-stick manoeuvre following the Essex Lopresti technique [6,7] and 6.5- $\mathrm{mm}$ cannulated screws were used for fixation of the tuberosity. Counter sinking was performed to avoid pressure over the skin and symptoms arising from this. 


\section{Cureus}
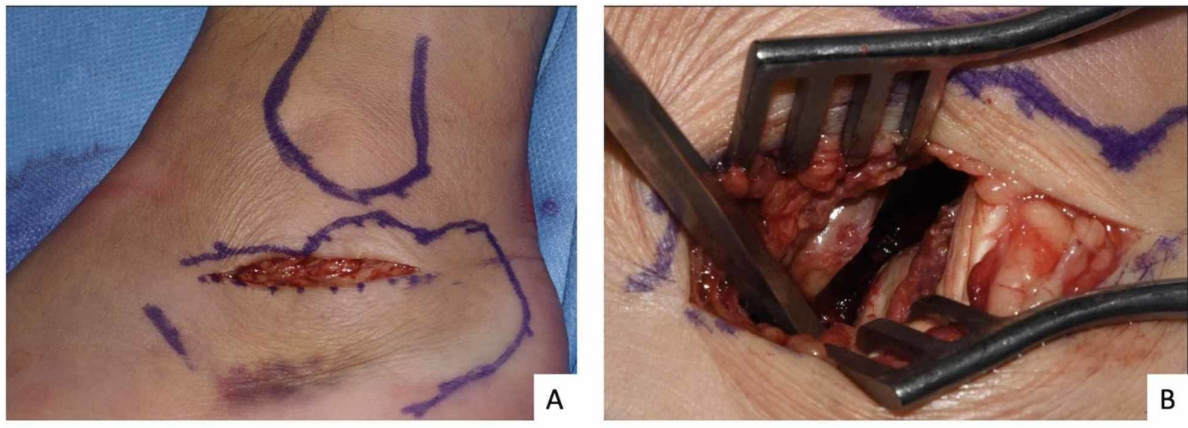

FIGURE 2: (A) Incision for MIS (limited sinus tarsi approach). (B) Restoration of the posterior facet using a small osteotome.

MIS, minimally invasive surgery

During the post-operative period, elevation of the operated limb was maintained along with nonweightbearing mobilisation. Patients were discharged home after wound check on the second post-operative day with below-knee lightweight cast. Further wound check was performed in the clinic weekly until the wound healed completely, whereas cast was continued for a total of six to eight weeks. The first follow-up after surgery did not always take place on the same day of the following week because of the difference in trauma list and fracture clinic appointments. Additional nurse lead wound review was arranged to record wound healing. Radiological assessment was performed while patients came for the removal of their casts at six weeks and then at three months and six months. Patients were followed up till radiological union was confirmed.

Both Bohler's and Gissane's angles were measured on lateral radiograph using PACS. Bohler's angle was measured by subtending two lines. The first line is drawn by connecting the highest points of anterior and posterior facets. The second line runs tangential to the superior edge of tuberosity (Figure $3 \mathrm{~A}$ ). An angle of 20 to 40 degrees is usually regarded as normal [5]. The Gissane's angle is an obtuse angle formed by the downward and upward slopes of the superior surface of the calcaneum measured on the lateral border directly inferior to the lateral process of the talus (Figure 3B). Its normal value is between 120 and 145 degrees [5].

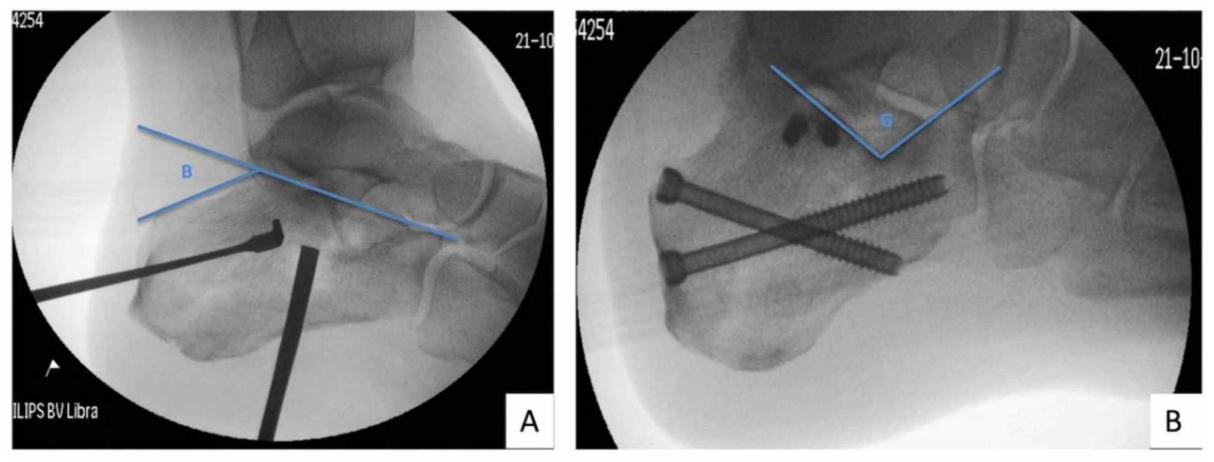

FIGURE 3: (A) Fluoroscopy-guided reduction of displaced posterior facet and restoration of Bohler's angle (B), as shown by blue lines. (B) Final position of MIS fixation and Gissane's angle (G), as shown by blue lines.

MIS, minimally invasive surgery

\section{Results}

A total of 39 calcanea (37 patients) were operated, of which 20 had open procedure and 19 had MIS procedure, including one bilateral surgery in each group. Bilateral surgery was performed on the same day for both sides in both groups. Of the 37 patients, 29 were males and 8 were females. Mean age of the patients in the MIS group was 42.18 years (range: 15-68 years) and that of the patients in the open group was 43 years (range: $21-75$ years). Of the fractures, 53.84\% $(n=21)$ were Sanders type III, 28.20\% $(n=11)$ were type 


\section{Cureus}

II and $17.94 \%(n=7)$ were type IV. Both groups had all three types of CFs (Table 1).

\begin{tabular}{|c|c|c|c|}
\hline & MIS $\left(n=18^{\star}\right)$ & ORIF ( $\left.=19^{\star}\right)$ & All patients $(n=37)$ \\
\hline Mean age, years (range) & $42.18(15-68)$ & $43(21-75)$ & 42.67 (15-75) \\
\hline \multicolumn{4}{|l|}{ Sex } \\
\hline Male & 13 & 16 & 29 \\
\hline Female & 5 & 3 & 8 \\
\hline \multicolumn{4}{|l|}{ Side } \\
\hline Right & 11 & 12 & 23 \\
\hline Left & 8 & 8 & 16 \\
\hline \multicolumn{4}{|c|}{ Sanders type, $n$ (percentage) } \\
\hline Type II & $3(15.78 \%)$ & $8(40 \%)$ & $11(28.20 \%)$ \\
\hline Type III & $11(57.89 \%)$ & $10(50 \%)$ & $21(53.84 \%)$ \\
\hline Type IV & $5(26.31 \%)$ & $2(10 \%)$ & 7 (17.94\%) \\
\hline
\end{tabular}

\section{TABLE 1: Demographic data and types of calcaneal fracture according to Sanders classification}

*One patient in each group underwent bilateral surgery.

MIS, minimally invasive surgery; ORIF, open reduction internal fixation

In the MIS group, Bohler's angle improved from a pre-operative mean of 7.7 degrees to 32.6 degrees and Gissane's angle improved from a pre-operative mean of 152.7 degrees to 136.3 degrees. In the open group, Bohler's angle improved from a pre-operative mean of 7.7 degrees to 31.5 degrees and Gissane's angle improved from a pre-operative mean of 155.8 degrees to 139.2 degrees. Mean corrections are detailed in Table 2. Further analysis of the cohort showed that patients over 60 years of age $(n=7)$ had significantly narrower post-operative calcaneal width in the MIS group.

\begin{tabular}{|c|c|c|c|}
\hline & MIS & ORIF & p-Value \\
\hline Heel height correction (mm) & 5.3 & 5.0 & 0.866 \\
\hline Heel width correction (mm) & -2.7 & -2.3 & 0.803 \\
\hline Bohler's angle correction & 24.9 & 23.8 & 0.811 \\
\hline Post-operative Bohler's angle & 32.6 & 31.5 & 0.713 \\
\hline Gissane's angle correction & -16.4 & -16.6 & 0.954 \\
\hline Post-operative Gissane's angle & 136.3 & 139.2 & 0.142 \\
\hline
\end{tabular}

TABLE 2: Comparison of mean surgical outcomes

MIS, minimally invasive surgery; ORIF, open reduction internal fixation

All fixations showed radiological evidence of union by six months follow-up, which was confirmed by plain radiograph. Neither open nor minimally invasive group resulted in any post-operative wound infections in our series. Mean hospital stay for the MIS group was four nights (range: 2-13 nights) and that of the open group was five nights (range: 2-17 nights).

The mean cost for implant used for open procedure was $£ 882.79$, and the implant cost per patient for MIS was $£ 142.89$ (Table 3). The difference was significant. Mean utilisation of cumulative X-ray dose was significantly higher in MIS (0.764 mGy) in comparison to the same in open surgery (0.392 mGy), as shown in 


\section{Cureus}

Table 4. The result was also statistically significant.

\begin{tabular}{|c|c|c|}
\hline & \multicolumn{2}{|c|}{ Cost of Implant (Pound Stirling) } \\
\hline & MIS & ORIF \\
\hline Mean & 142.90 & 882.79 \\
\hline Min-Max & 39.42-236.52 & $780.30-1174.50$ \\
\hline Standard deviation & 58.92 & 96.07 \\
\hline P-value & $<0.001$ & \\
\hline
\end{tabular}

TABLE 3: Difference in cost of implant used in MIS and open calcaneal fracture surgery

MIS, minimally invasive surgery; ORIF, open reduction internal fixation

\begin{tabular}{|c|c|c|}
\hline & \multicolumn{2}{|c|}{ Cumulative X-Ray Dose (in mGy) } \\
\hline & MIS & ORIF \\
\hline Mean & 0.764 & 0.392 \\
\hline Standard deviation & 0.53 & 0.16 \\
\hline P-value & 0.027 & \\
\hline
\end{tabular}

TABLE 4: Difference in utilisation of cumulative X-ray dose in MIS and ORIF

MIS, minimally invasive surgery; ORIF, open reduction internal fixation

Average surgical time for MIS was 64.9 minutes and that of open surgery was 106.3 minutes. Average waiting time for MIS was 6.6 days and that for ORIF was 9.8 days. Wound healing was quicker (average 13.4 days) in MIS than ORIF (average 17.2 days). All these differences were statistically significant (Table 5).

\begin{tabular}{|c|c|c|c|c|c|}
\hline & \multicolumn{2}{|l|}{ MIS } & \multicolumn{2}{|l|}{ ORIF } & \multirow{2}{*}{ p-Value } \\
\hline & Min-Max & Average & Min-Max & Average & \\
\hline Surgical time (minutes) & $33-90$ & 64.9 & 65-145 & 106.3 & $<0.001$ \\
\hline Time to surgery (days) & $0-13$ & 6.6 & 4-16 & 9.8 & 0.025 \\
\hline Wound healing time (days) & $10-16$ & 13.4 & $14-21$ & 17.2 & \\
\hline
\end{tabular}

TABLE 5: Surgical timings and wound healing comparison

MIS, minimally invasive surgery; ORIF, open reduction internal fixation

\section{Discussion}

Both operative and conservative treatment have been used for displaced intra-articular CFs. Various surgical techniques have been described. These include the lateral extensile "L" approach, MIS sinus tarsi approach, percutaneous approach, double external fixator distraction technique, combination approach, balloonassisted calcaneoplasty, subtalar arthroscopy assisted fixation and arthrodesis [3,8-12]. No evidence for a gold standard treatment has been reported.

The distribution of cases in our study revealed that type II Sanders was about three times more in the ORIF group, whereas type IV Sanders was about 2.5 times more in the MIS group (Table 1). Further analysis of the 
cohort showed that most of the ORIFs ( $\mathrm{n}=18.90 \%$ ) were performed by 2016 and only five MIS (around 26\%) were performed during this period. In our centre, most of the calcaneal surgery after 2016 was performed following MIS technique. Interestingly, Griffin et al. published their report of a multicentre Randomised Control Trial, UK Heel Fracture Trial, in 2014 [13]. The finding of this trial has shown no symptomatic or functional advantage of operative outcome in comparison to non-operative treatment. It has also reported high risk of complication and re-operation following calcaneal surgery; hence, they recommended against it. It appears that in our centre, type II and type III Sanders fractures were treated by MIS as the technique got popularity over ORIF during this period.

The extensile lateral approach has reported good to excellent clinical outcome in $60-85 \%$ of cases [14]. Open surgery has been associated with high rates of wound complications. In some series, it has been up to $25 \%$, where $21 \%$ of them required surgical treatment [2].

Wang et al. included 492 CFs and looked into the functional outcome of open and MIS procedure [1]. They did not find significant difference in outcome, which is supported by our radiological findings in both groups. Another similar comparative study by Weber et al. included 24 calcaneum operated by limited open reduction and 26 calcaneum was operated by conventional extensile lateral approach [12]. In this study, there was no wound complication in the MIS group and only four minor complications in open surgical group. Several other case series have shown positive outcome with MIS for displaced intra-articular calcaneum fracture where arthroscopic assisted reduction was performed [8-10].

In our cohort of open surgery, Bohler's angle improved from a pre-operative mean of 7.7 degrees to 31.5 degrees (average correction: 23.8 degrees) and Gissane's angle improved from a pre-operative mean of 155.8 degrees to 139.2 degrees (average correction: 16.6 degrees). We could not find any statistically significant difference irrespective of types of fracture as our patients were equally distributed in both groups. This result is comparable to the one published by Jain et al. where the mean Bohler's angle improved from 4.15 degrees to 25.47 degrees and mean Gissane's angle improved from 151 degrees to 121 degrees [14]. A small cohort $(n=7)$ of our patients over 60 years of age has been found to have a significant correction of calcaneal width after MIS $(n=4$; average heel width correction of $4.5 \mathrm{~mm})$ in comparison to ORIF $(n=3$; average heel width correction of $2.3 \mathrm{~mm}$ ). In over 60 years, MIS might be a better option than open surgery, although it will require a study with a larger number to reach a conclusion.

No study was found looking into radiation dose and wound healing time in CF surgery. Our study has compared those factors in both the ORIF and MIS groups. In our series, the open group required significantly longer ( $\mathrm{p}<0.001$ ) time for wound healing in comparison to the MIS group (Table 5). This is due to the larger incision and more dissection required in extensile approach.

In our cohort of open surgery, Zimmer Biomet A.L.P.S. calcaneal plates with variable number (6-15) locking screws were used. In the MIS group, two to six cannulated screws were used. Price of each A.L.P.S. plate was $£ 583.20$ and that of each screw was $£ 39.42$. We found a significant difference $(p<0.001)$ in implant cost in those two groups. We have only taken the cost of implants into consideration, although inclusion of prolonged hospital stay, more instruments and theatre equipment will certainly make the difference even pronounced. In our series, patients in the open group stayed one extra night in hospital on an average. A study by Clement et al. has shown significantly higher costs in open procedure comparing to other treatment [15]. MIS was found to be the least expensive followed by conservative and open surgery.

Our study is the first study looking at radiation dosage in different modalities of CFs. We noticed utilisation of significantly higher $(\mathrm{p}=0.027)$ fluoroscopic time and hence cumulative radiation dose in MIS. The mean cumulative radiation dose required for MIS was $0.764 \mathrm{mGy}$ and that for and open procedure was $0.392 \mathrm{mGy}$. In other words, double radiation dose was required in MIS on an average. It is similar to being exposed to 2.5 more standard chest X-rays, which require a mean entrance surface dose of $0.15 \mathrm{mGy}$ [16]. This is most likely due to the higher requirement of X-ray guidance for articular reduction with limited surgical exposure. This finding should remind us of the As Low As Reasonably Possible (ALARA) radiation safety principle [17]. It might be useful to bear in mind, regardless of procedure concerned, that the maximum annual dose limit is $20 \mathrm{mSv}$ for the body, $150 \mathrm{mSv}$ for thyroid and eyes and $900 \mathrm{mSv}$ for the hands [18], especially when only $24 \%$ of surgical trainees are reported to use a thyroid shield [19].

We understand that our study population was small, and studies including a larger number of patients would be required to conclude whether one method is superior than the other. Another limitation of our study is not to use any patient-reported outcome measures, as our aim was to find out radiological outcome in the first place. Assessment of a wider range of clinical outcomes, e.g., using the Manchester-Oxford Foot Questionnaire, would be useful to find the overall clinical outcome. We recommend multicentred randomised controlled trials with a large number of populations for comparative study among MIS and nonoperative management.

\section{Conclusions}

Minimally invasive calcaneal surgery is cheaper and quicker and can safely be performed early. It is 
associated with early wound healing and can achieve equally good radiological correction. We recommend evaluating every case on its own merit and to consider MIS while deciding on the surgical management of CFs.

\section{Additional Information \\ Disclosures}

Human subjects: Consent was obtained by all participants in this study. Animal subjects: All authors have confirmed that this study did not involve animal subjects or tissue. Conflicts of interest: In compliance with the ICMJE uniform disclosure form, all authors declare the following: Payment/services info: All authors have declared that no financial support was received from any organization for the submitted work. Financial relationships: All authors have declared that they have no financial relationships at present or within the previous three years with any organizations that might have an interest in the submitted work. Other relationships: All authors have declared that there are no other relationships or activities that could appear to have influenced the submitted work.

\section{References}

1. Wang Q, Li X, Sun Y, Yan L, Xiong C, Wang J: Comparison of the outcomes of two operational methods used for the fixation of calcaneal fracture. Cell Biochem Biophys. 2015, 72:191-196. 10.1007/s12013-014-0436-0

2. Tufescu TV, Buckley R: Age, gender, work capability, and worker's compensation in patients with displaced intraarticular calcaneal fractures. J Orthop Trauma. 2001, 15:275-279. 10.1097/00005131-200105000-00007

3. Folk JW, Starr AJ, Early JS: Early wound complications of operative treatment of calcaneus fractures: analysis of 190 fractures. J Orthop Trauma. 1999, 13:369-372. 10.1097/00005131-199906000-00008

4. Dhillon MS, Prabhakar S: Controversies in calcaneus fracture management: a systematic review of the literature. Musculoskelet Surg. 2011, 95:171-181. 10.1007/s12306-011-0114-y

5. Sanders R: Current concepts review: displaced intra-articular fractures of the calcaneus . J Bone Jt Surg. 2000, 82:225-250. 10.2106/00004623-200002000-00009

6. Essex-Lopresti P: The mechanism, reduction technique, and results in fractures of the OS calcis . Br J Surg. 1952, 39:395-419. 10.1002/bjs.18003915704

7. Shih JT, Kuo CL, Yeh T Te, Shen HC, Pan RY, Wu CC: Modified Essex-Lopresti procedure with percutaneous calcaneoplasty for comminuted intra-articular calcaneal fractures: a retrospective case analysis. BMC Musculoskelet Disord. 2018, 19:1-6. 10.1186/s12891-018-1995-9

8. Rammelt S, Amlang M: Percutaneous treatment of less severe intraarticular calcaneal fractures . Clin Orthop Relat Res. 2009, 468:983-990. 10.1007/s11999-009-0964-x

9. Potter BMQ, Nunley JA: Long-term functional outcomes after operative treatment for intra-articular fractures of the calcaneus. J Bone Joint Surg Am. 2009, 91:1854-1860. 10.2106/JBJS.H.01475

10. Stulik J, Stehlik J, Rysavy M, Wozniak A: Minimally-invasive treatment of intra- articular fractures of the calcaneum. J Bone Jt Surg Br. 2006, 88:1634-1641. 10.1302/0301-620X.88B12.17379

11. Schuberth JM, Cobb MD, Talarico RH: Minimally invasive arthroscopic-assisted reduction with percutaneous fixation in the management of intra-articular calcaneal fractures: a review of 24 cases. J Foot Ankle Surg. 2009, 48:315-322. 10.1053/j.jfas.2009.01.002

12. Weber M, Lehmann O, Sägesser D, Krause F: Limited open reduction and internal fixation of displaced intra-articular fractures of the calcaneum. J Bone Joint Surg Br. 2008, 90:1608-1616. 10.1302/0301620X.90B12.20638

13. Griffin D, Parsons N, Shaw E, Kulikov Y, Hutchinson C, Thorogood M, Lamb SE: Operative versus nonoperative treatment for closed, displaced, intra-articular fractures of the calcaneus: randomised controlled trial. BMJ. 2014, 349:4483. 10.1136/bmj.g4483

14. Jain S, Jain AK, Kumar I: Outcome of open reduction and internal fixation of intraarticular calcaneal fracture fixed with locking calcaneal plate. Chin J Traumatol. 2013, 16:355-360. 10.3760/cma.j.issn.10081275.2013.06.008

15. Clement RC, Lang PJ, Pettett BJ, Overman RA, Ostrum RF, Tennant JN: Sanders II/III calcaneus fractures in laborers: a cost-effectiveness analysis and call for effectiveness. J Orthop Trauma. 2017, 31:299-304. 10.1097/BOT.0000000000000813

16. Hart D, Hillier MC, Wall BF: Doses to patients from medical X-ray examinations in the UK - 2000 review . NRPB. 2002, 2002:1-66.

17. Kaplan DJ, Patel JN, Liporace FA, Yoon RS: Intraoperative radiation safety in orthopaedics: a review of the ALARA (As low as reasonably achievable) principle. Patient Saf Surg. 2016, 10:27. 10.1186/s13037-0160115-8

18. Lindell B, Dunster HJ, Valentin J: International Commission on Radiological Protection: history, policies, procedures. ICRP. 1991, 21:1-12.

19. Khan F, Javed A, Rauf S: Awareness and attitudes amongst basic surgical trainees regarding radiation in orthopaedic trauma surgery. Biomed Imaging Interv J. 2010, 6:25. 10.2349/biij.6.3.e25 